\title{
O VÍNCULO NO TRATAMENTO DA TUBERCULOSE NA ATENÇÃO PRIMÁRIA À SAÚDE: UMA REVISÃO INTEGRATIVA
}

\author{
The bond in the treatment of tuberculosis in Primary Health Care: an integrative \\ review
}

\section{El vínculo durante el tratamiento de la tuberculosis en la Atención Primaria de Salud: una revisión integrativa}

\section{Melisane Regina Lima Ferreira iD}

Fundação Universidade Federal de Rondônia - UNIR - Porto Velho (RO) - Brasil

\author{
Alexsandra Araújo Santos iD \\ Fundação Universidade Federal de Rondônia - UNIR - Porto Velho (RO) - Brasil
}

Nathalia Halax Orfão iD

Fundação Universidade Federal de Rondônia - UNIR - Porto Velho (RO) - Brasil

\section{RESUMO}

Objetivo: Descrever como o vínculo tem contribuído (ou não) para o manejo do cuidado da tuberculose (TB) na Atenção Primária à Saúde (APS). Métodos: Trata-se de uma revisão integrativa da literatura, realizada nas bases de dados Literatura LatinoAmericana e do Caribe em Ciências da Saúde (LILACS), Literatura Internacional em Ciências da Saúde (MEDLINE), Cumulative Index to Nursing and Allied Health Literature (CINAHL), Scopus e Web of Science, a partir de artigos científicos completos e em livre acesso, publicados no período entre 2009 a 2019, nos idiomas português, inglês e espanhol, bem como aqueles que, em seus resultados, abordassem aspectos relacionados à temática do estudo. Resultados: Selecionaram-se 151 artigos nas bases de dados, dos quais se excluíram 134 por não atenderem aos critérios previamente estabelecidos, contabilizando, ao final, 17 artigos científicos para serem lidos e analisados na íntegra. Identificaram-se elementos potencializadores e dificultadores para a construção, estabelecimento e fortalecimento do vínculo no manejo da TB na APS. Conclusão: Esta revisão aponta a necessidade de novas discussões acerca da temática, por meio da identificação dos pontos de estrangulamento na organização e desempenho dos serviços de saúde, objetivando a implementação de ações integradas e intersetoriais, a partir da compreensão de aspectos que dificultam a reorientação de práticas e ofertas de serviços, tendo em vista a construção de um novo paradigma de cuidado aos doentes de TB.

Descritores: Tuberculose; Acolhimento; Relações Profissional-Paciente; Pesquisa sobre Serviços de Saúde; Atenção Primária à Saúde.

\section{ABSTRACT}

Objective: To describe how the bond has contributed (or not) to the management of Tuberculosis (TB) care in Primary Health Care (PHC). Methods: This is an integrative review of the literature, carried out in the Latin American and Caribbean Health Sciences (LILACS), International Literature in Health Sciences (MEDLINE), Cumulative Index to Nursing and Allied Health Literature (CINAHL), Scopus and Web of Science databases, from complete scientific articles and open access, published between 2009-2019 in Portuguese, English, and Spanish languages, as well as those that, in their results, addressed aspects related to the thematic of the study. Results: 151 articles were selected in the databases, of which 134 were excluded because they did not meet the previously established criteria, and 17 scientific articles were chosen at the end to be read and analyzed in their entirety. Potentiating and hindering elements were identified for the construction, establishment, and strengthening of the bond in the management of TB in PHC. Conclusion: This review points out the need for new discussions on the subject, through the identification of bottlenecks in the organization and performance of health services, aiming at the implementation of integrated and intersectoral actions, from the understanding of aspects that make it difficult to reorientation practices and service offerings, intending to build a new paradigm of care for TB patients.

Descriptors: Tuberculosis; User Embracement; Professional-Patient Relations; Health Services Research; Primary Health Care.

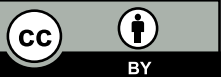




\section{RESUMEN}

Objetivo: Describir cómo el vínculo ha contribuido (o no) para el manejo del cuidado de la tuberculosis (TB) en la Atención Primaria de Salud (APS). Métodos: Se trata de una revisión integrativa de la literatura realizada en las bases de datos Literatura Latino-Americana y del Caribe en Ciencias de la Salud (LILACS), Literatura Internacional en Ciencias de la Salud (MEDLINE), Cumulative Index to Nursing and Allied Health Literature (CINAHL), Scopus y Web of Science a partir de artículos cientificos completos y de libre acceso publicados en el período entre 2009 y 2019 en los idiomas portugués, inglés y español así como aquellos que incluyeran en sus resultados los aspectos relacionados con la temática del estudio. Resultados: Se han elegido 151 artículos de las bases de datos de los cuales 134 han sido excluidos por el no cumplimiento de los criterios establecidos lo que llevó a un total final de 17 artículos científicos para la lectura y el análisis del texto completo. Se han identificado los elementos facilitadores y dificultadores de la construcción, el establecimiento y el fortalecimiento del vínculo para el manejo de la TB en la APS. Conclusión: Esa revisión señala la necesidad de nuevas discusiones sobre el tema a través de la identificación de los puntos de estrangulación de la organización y el rendimiento de los servicios de salud con el objetivo de implementar acciones integradas e intersectoriales a partir de la comprensión de los aspectos que dificultan la reorientación de prácticas y ofertas de servicios para la construcción de un nuevo paradigma de cuidado para los enfermos de TB.

Descriptores: Tuberculosis; Acogimiento; Relaciones Profesional-Paciente; Investigación sobre Servicios de Salud; Atención Primaria de Salud.

\section{INTRODUÇÃO}

A tuberculose (TB) persiste como um grave problema de saúde pública, contribuindo para o quadro de desigualdade e exclusão social ${ }^{(1)}$. Mesmo sendo tratável e curável, ainda se observam desfechos desfavoráveis no tratamento, como o óbito, favorecendo a posição de primeira causa de morte entre as doenças infectocontagiosas no mundo(2) e o abandono do tratamento, considerado um dos aspectos mais desafiantes para o controle da doença $a^{(3)}$.

Por possuir o papel de ordenar e coordenar o cuidado nas Redes de Atenção à Saúde (RAS) para condições crônicas, tal como a TB, cabe aos serviços da Atenção Primária à Saúde (APS) a responsabilidade pela articulação e fortalecimento do vínculo com a população de sua área adscrita. Além disso, por ser considerada como porta de entrada preferencial do usuário no sistema de saúde, deve ser capaz de atuar no manejo das fragilidades de resolubilidade das ações para o controle da doença ${ }^{(4,5)}$.

A longitudinalidade é um dos atributos essenciais da APS e relaciona-se com o estabelecimento de vínculo e corresponsabilidade nas relações entre profissionais/equipe/unidade de saúde e usuários/família/comunidade, independentemente da presença de agravos. Uma vez que o acolhimento é fundamental para a criação de vínculo, fragilidades no acesso aos serviços de saúde, no diagnóstico, na estigma perante a doença, no tratamento e no acompanhamento por parte dos profissionais afetam esse atributo, limitando o desempenho e a oferta dos serviços de saúde ${ }^{(6-8)}$.

O vínculo é uma das dimensões que permite analisar como vem sendo realizado o manejo da TB na APS. Torna-se, portanto, uma ferramenta essencial para a promoção da saúde e prevenção da doença, bem como para a adesão e continuidade do tratamento. Somado à educação em saúde, constituem instrumentos fundamentais para o acompanhamento da TB, por proporcionarem a troca de diálogo e autonomia no decorrer do tratamento ${ }^{(9)}$, influenciando diretamente no sucesso do desfecho e controle do agravo(3).

No âmbito da saúde coletiva, a análise dos elementos potencializadores e dificultadores para a construção, estabelecimento e manutenção do vínculo auxiliam quanto ao desenvolvimento de políticas públicas, habilidades individuais e coletivas e reorientação de serviços visando à promoção em saúde, a fim de garantir a efetividade desse atributo essencial na APS.

Portanto, levando em conta a necessidade da inclusão de uma abordagem humanizada entre profissional de saúde e usuário, por meio da escuta de saberes, dúvidas, angústias e identificação de vulnerabilidades, esta revisão tem como objetivo descrever como o vínculo tem contribuído (ou não) para o manejo do cuidado da TB na APS.

\section{MÉTODOS}

Trata-se de uma revisão integrativa da literatura, realizada em maio de 2019, por meio de um levantamento nas bases de dados Literatura Latino-Americana e do Caribe em Ciências da Saúde (LILACS), Literatura Internacional em 
Ciências da Saúde (MEDLINE), Cumulative Index to Nursing and Allied Health Literature (CINAHL), Scopus e Web of Science, visando a identificar e sintetizar os estudos científicos divulgados no cenário nacional e internacional.

Para nortear esta revisão, formulou-se a seguinte questão norteadora: "como o vínculo tem contribuído (ou não) para o manejo do cuidado da TB na APS?". Para isso, utilizaram-se as expressões de busca por meio da associação dos descritores indexados no Descritores em Ciências da Saúde (DeCS) e no Medical Subject Headings (MESH): "tuberculose", "acolhimento", "relações profissional-paciente", "pesquisa sobre serviços de saúde", "avaliação de serviços de saúde", "Atenção Primária à Saúde" e "Programa Saúde da Família", com a utilização dos booleanos "AND" e "OR" (Figura 1).

Tuberculose AND "Relações Profissional-Paciente" AND ("Pesquisa sobre serviços de saúde" OR "Avaliação de Serviços de Saúde")

Tuberculose AND "Pesquisa sobre serviços de saúde" OR

"Avaliação de Serviços de Saúde") AND ("Atenção Primária à Saúde" OR "Programa Saúde da Família")

Tuberculose AND Acolhimento AND (“Atenção Primária à Saúde" OR "Programa Saúde da Família")

Tuberculose AND "Serviços de Saúde" AND "Relações Profissional Paciente"
Lilacs - 1

Medline - 5

CINAHL - 0

Scopus - 0

Web of Science - 0

Lilacs - 3

Medline - 27

CINAHL - 6

Scopus - 28

Web of Science - 2

Lilacs - 9

Medline - 1

CINAHL - 0

Scopus - 3

Web of Science - 2

Lilacs - 2

Medline - 18

CINAHL - 19

Scopus - 24

Web of Science - 1

Figura 1 - Expressões de busca e resultados do processo de seleção de artigos científicos, de acordo com cada base de dados utilizada.

A seleção dos estudos envolveu duas pesquisadoras independentes, havendo a possibilidade de uma terceira pesquisadora, caso houvesse discordância na seleção dos artigos. Como critérios de inclusão, optou-se por artigos científicos completos, em open acess, publicados no período entre 2009 a 2019, nos idiomas português, inglês e espanhol, bem como aqueles que, em seus resultados, abordassem aspectos relacionados à temática do estudo. Foram excluídos os artigos duplicados, artigos de revisão, teses, dissertações, monografias, editoriais, artigos de reflexão ou de debates, cartas e manuais.

A categorização do estudo e a análise dos resultados encontrados será apresentada em um quadro contendo os autores, ano/local do estudo e os elementos potencializadores e dificultadores para o vínculo no manejo da TB.

\section{RESULTADOS}

Mediante a busca nas bases de dados, encontraram-se 151 artigos, sendo excluídos 134 artigos - 82 por não estarem em livre acesso, 25 por estarem duplicados, 15 por não discutirem aspectos relacionados à temática do estudo, oito por estarem fora do período previamente selecionado, um por não estar completo, um por estar em francês, um por ser uma tese e mais um por ser revisão de literatura.

Portanto, após a busca na literatura pelos revisores pares, selecionaram-se, ao final, 17 artigos científicos para serem lidos e analisados na íntegra (Figura 2). 


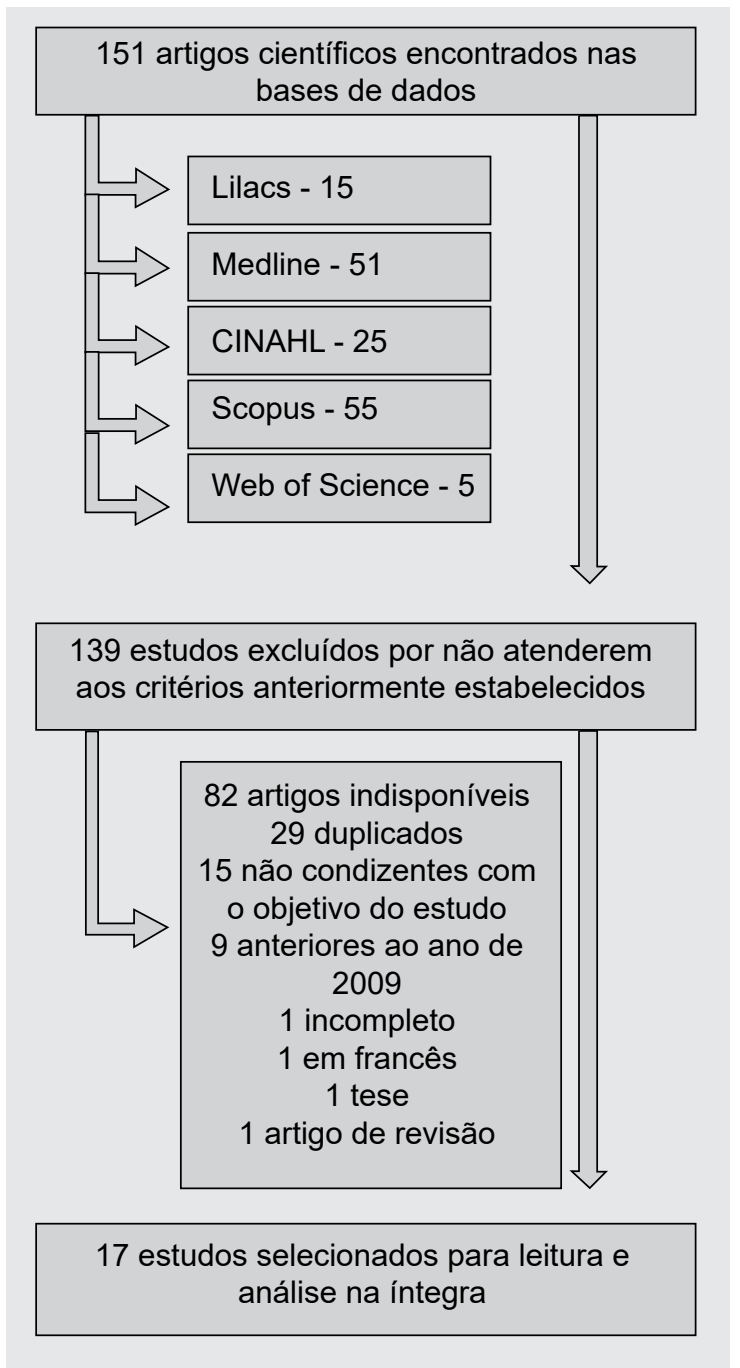

Figura 2 - Etapas do processo de seleção de artigos científicos para a revisão integrativa da literatura.

A maioria dos artigos selecionados estava no idioma português (52,9\%), havia sido publicados em 2017 (23,5\%) e produzidos no cenário nacional (82,3\%), sendo o estado de São Paulo e da Paraíba os de maior produção, com cinco e três estudos, respectivamente, o que demonstra o crescente interesse na temática da avaliação das dimensões da APS, em especial o vínculo, uma vez que se constitui como um elemento estruturante na atenção e controle da TB(10), contribuindo para o aprimoramento da organização e do desempenho dos serviços de saúde.

Houve predominância de estudos com abordagem quantitativa (82,3\%). Quanto ao tipo de estudo, observou-se pesquisas descritivas $(35,4 \%)$, operacionais $(17,8 \%)$, etnográficas, avaliativas, transversais e ecológicas $(11,7 \%)$. $\mathrm{E}$, em relação à classificação dos estudos de acordo com o nível de evidência, adotando a proposta por Melnyk e Fineout-Overholt(11), verificou-se que todos apresentaram força de evidência VI, que se refere a único estudo descritivo ou qualitativo.

A maioria dos estudos identifica que se constituem como elementos potencializadores e que contribuem para o vínculo no manejo da TB: as orientações a respeito da doença, do tratamento, de efeitos colaterais e de outros problemas de saúde; ser atendido pelos mesmos profissionais; educação em saúde; a operacionalização do tratamento diretamente observado (TDO); as visitas domiciliares (VD); capacitações e treinamentos da equipe de saúde interdisciplinar; a família e rede de apoio social; a oferta de insumos e incentivos, a importância dos agentes comunitários de saúde (ACS); bem como a articulação com outros serviços de saúde (Quadro I).

O estigma da doença; problemas sociais e psicológicos durante o tratamento, tais como o uso de drogas e álcool; dificuldades no acesso ao diagnóstico e ao tratamento da TB; desestruturação; fragmentação dos serviços; e falhas no sistema de referência e contrarreferência são citados como elementos dificultadores e que não contribuem para o vínculo no manejo da TB (Quadro I). 
Quadro I - Resultados dos artigos desta revisão, de acordo com os autores, ano, local do estudo e os elementos potencializadores e dificultadores para o vínculo no manejo da tuberculose.

\begin{tabular}{|c|c|c|c|}
\hline \multirow{2}{*}{ Autor } & \multirow{2}{*}{$\begin{array}{l}\text { Ano/ Local } \\
\text { do Estudo }\end{array}$} & \multicolumn{2}{|c|}{ Vínculo no manejo da tuberculose } \\
\hline & & Elementos potencializadores & Elementos dificultadores \\
\hline Neves et al..$^{(3)}$ & 2016/ Brasil & $\begin{array}{l}\text { Ser atendido pelos mesmos profissionais; } \\
\text { compreensão, clareza e esclarecimento das } \\
\text { dúvidas dos doentes de TB pelos profissionais; } \\
\text { proximidade entre a residência do doente e a } \\
\text { unidade de saúde; acolhimento pela equipe } \\
\text { de saúde. }\end{array}$ & $\begin{array}{l}\text { Dificuldades de acesso ao diagnóstico e } \\
\text { tratamento; priorização dos sinais/sintomas da } \\
\text { TB em detrimento de outros problemas de saúde; } \\
\text { falta de VD; longa espera para atendimento nas } \\
\text { unidades de saúde. }\end{array}$ \\
\hline $\begin{array}{l}\text { Pinheiro et } \\
\text { al. }{ }^{\left({ }^{6}\right)}\end{array}$ & 2017/ Brasil & $\begin{array}{l}\text { Responsabilização pela saúde do conjunto } \\
\text { de pessoas que vivem em determinada área } \\
\text { adscrita, independente da ausência ou presença } \\
\text { da doença. }\end{array}$ & $\begin{array}{l}\text { Preconceito por parte dos profissionais; } \\
\text { dificuldades de acesso à realização de exames, } \\
\text { consultas e tratamento, bem como falhas no } \\
\text { sistema de referência e contra referência. }\end{array}$ \\
\hline Alves et al. ${ }^{(7)}$ & 2016/ Brasil & $\begin{array}{l}\text { Orientações para diminuir as dúvidas e receios } \\
\text { quanto à doença; necessidade constante de } \\
\text { atualizações e capacitações dos profissionais } \\
\text { de saúde; ampliação dos atendimentos } \\
\text { descentralizados; implementação do TDO, } \\
\text { fortalecimento do trabalho em equipe e } \\
\text { otimização da referência e contra referência. }\end{array}$ & $\begin{array}{l}\text { Falta de confiança nos profissionais pelos } \\
\text { doentes de TB para explicar de forma correta } \\
\text { os procedimentos, passando segurança em suas } \\
\text { palavras. }\end{array}$ \\
\hline $\begin{array}{l}\text { Andrade } \\
\text { et al. } .^{(9)}\end{array}$ & 2016/ Brasil & $\begin{array}{l}\text { TDO; autocuidado em saúde e suporte na } \\
\text { educação em saúde; apoio e participação } \\
\text { familiar. }\end{array}$ & $\begin{array}{l}\text { Predominância de um modelo de saúde } \\
\text { fragmentado; Falta de envolvimento com grupos } \\
\text { de apoio e profissionais especializados como } \\
\text { psicólogos e assistentes sociais na própria } \\
\text { unidade de saúde para acolher o doente de TB; } \\
\text { uso de drogas e álcool. }\end{array}$ \\
\hline $\begin{array}{c}\text { Figueiredo et } \\
\text { al. }^{(10)}\end{array}$ & 2011/ Brasil & $\begin{array}{l}\text { Ser atendido pelo mesmo profissional; } \\
\text { informações pelos profissionais, sobre o correto } \\
\text { uso da terapêutica, possíveis efeitos colaterais e } \\
\text { riscos; operacionalização do TDO; capacitação } \\
\text { dos profissionais de saúde. }\end{array}$ & $\begin{array}{l}\text { Caráter estigmatizante da doença; transtornos } \\
\text { emocionais como depressão, agressividade e } \\
\text { isolamento }\end{array}$ \\
\hline $\begin{array}{l}\text { Lewis, } \\
\text { Newell(12) }\end{array}$ & 2009/ Nepal & $\begin{array}{l}\text { Equipe de saúde acessível e solidária para } \\
\text { discutir problemas que surgem durante o } \\
\text { tratamento; flexibilidade no tratamento para } \\
\text { que atividades básicas continuem; melhoria } \\
\text { da comunicação; educação em saúde sobre } \\
\text { TB com a família do doente. }\end{array}$ & $\begin{array}{l}\text { Aspectos sociais e psicológicos durante o } \\
\text { tratamento; falta de informações ao doente e à } \\
\text { família sobre a doença, tratamento e possíveis } \\
\text { efeitos colaterais; sensação de isolamento pela } \\
\text { família; estigma da doença pela comunidade } \\
\text { em geral. }\end{array}$ \\
\hline \begin{tabular}{c|} 
Chhea, \\
Warren, \\
Manderson $^{(13)}$
\end{tabular} & $\begin{array}{c}2010 / \\
\text { Camboja }\end{array}$ & $\begin{array}{l}\text { Segurança e reconhecimento no trabalho, } \\
\text { oportunidade de capacitação, ambientes } \\
\text { de trabalho flexíveis e subsídios para os } \\
\text { profissionais de saúde. }\end{array}$ & $\begin{array}{l}\text { Fragmentação no acesso ao diagnóstico e } \\
\text { tratamento. }\end{array}$ \\
\hline $\begin{array}{l}\text { Ponce et } \\
\text { al. }^{(14)}\end{array}$ & 2011/Brasil & $\begin{array}{l}\text { Ser atendido pelo mesmo profissional; } \\
\text { comunicação eficaz com enfoque para a } \\
\text { subjetividade do doente; informações sobre o } \\
\text { esquema terapêutico. }\end{array}$ & Não informado \\
\hline $\begin{array}{l}\text { Rocha, } \\
\text { Adorno(15) }^{(15)}\end{array}$ & 2012/ Brasil & $\begin{array}{l}\text { Importância dos ACS pelo contato em âmbito } \\
\text { familiar e maior abertura para ouvir os } \\
\text { desconfortos e angústias dos doentes; família ou } \\
\text { rede de apoio social; monitoramento frequente, } \\
\text { apoio, compreensão diante das dificuldades } \\
\text { enfrentadas, condições e informações } \\
\text { acessíveis. }\end{array}$ & $\begin{array}{l}\text { Distanciamento imposto socialmente entre o } \\
\text { doente e o profissional; falhas nas orientações } \\
\text { sobre a doença e seu tratamento; desestruturação } \\
\text { dos serviços de saúde; falta de estabelecimento } \\
\text { de uma relação de confiança e reciprocidade; } \\
\text { preocupação apenas com a medicalização e } \\
\text { características clínicas e epidemiológicas dos } \\
\text { doentes; postura e autoritarismo dos profissionais } \\
\text { de saúde; uso de drogas e álcool; estigma da } \\
\text { doença. }\end{array}$ \\
\hline $\begin{array}{c}\text { Marquieviz et } \\
\text { al. }^{(16)}\end{array}$ & 2013/ Brasil & $\begin{array}{l}\text { Treinamentos e capacitação dos profissionais } \\
\text { de saúde quanto ao manejo da TB; apoio às } \\
\text { questões sociais associadas. }\end{array}$ & Não informado \\
\hline
\end{tabular}




\begin{tabular}{|c|c|c|c|}
\hline $\begin{array}{l}\text { Kielmann et } \\
\text { al. }^{(17)}\end{array}$ & 2014/ Índia & $\begin{array}{l}\text { Estabelecimento de confiança como um } \\
\text { elemento crucial que sustenta a colaboração } \\
\text { entre os atores; TDO. }\end{array}$ & Não informado \\
\hline $\begin{array}{l}\text { Souza et } \\
\text { al. }{ }^{(18)}\end{array}$ & 2014/ Brasil & $\begin{array}{l}\text { Serviços acessíveis, custo efetivo, funcionando } \\
\text { integrados e fundamentados nas necessidades } \\
\text { de saúde da pessoa, família e comunidade; } \\
\text { enfermagem na condução do TDO; trabalho em } \\
\text { equipe interdisciplinar; papel do ACS. }\end{array}$ & $\begin{array}{l}\text { Drogadição, etilismo, crises familiares e outros } \\
\text { problemas sociais; insegurança no vínculo } \\
\text { trabalhista e poucos espaços para qualificação } \\
\text { na perspectiva da Educação Permanente em } \\
\text { Saúde pelos profissionais de saúde. }\end{array}$ \\
\hline $\begin{array}{l}\text { Balderrama et } \\
\text { al. }^{(19)}\end{array}$ & 2014/ Brasil & $\begin{array}{l}\text { Estrutura das unidades de saúde; maior } \\
\text { tempo de trabalho e menor rotatividade dos } \\
\text { profissionais de saúde; oferta de insumos; fluxo } \\
\text { laboratorial adequado; capacitação contínua dos } \\
\text { profissionais de saúde. }\end{array}$ & $\begin{array}{l}\text { Sobrecarga de trabalho dos profissionais de } \\
\text { saúde. }\end{array}$ \\
\hline $\begin{array}{l}\text { Clementino, } \\
\text { Miranda }^{(20)}\end{array}$ & 2015/ Brasil & $\begin{array}{l}\text { Unidade de Saúde da Família como porta de } \\
\text { entrada, promotora do acesso aos usuários, } \\
\text { com diagnóstico precoce da TB e supervisão } \\
\text { do tratamento por intermédio da VD; escuta } \\
\text { solidária; trabalho conjunto entre o doente e seus } \\
\text { familiares; organização da equipe de saúde. }\end{array}$ & $\begin{array}{l}\text { Dificuldade de acesso; dificuldades econômica } \\
\text { e social; estigma da doença; foco de atenção à } \\
\text { saúde por parte dos profissionais ainda centrado } \\
\text { no saber médico; praticamente inexistindo espaço } \\
\text { para o diálogo e construção do conhecimento; } \\
\text { distanciamento dos profissionais de saúde de } \\
\text { seu papel de educador. }\end{array}$ \\
\hline $\begin{array}{l}\text { Furlan, } \\
\text { Santos } \\
\text { Júnior, } \\
\text { Marcon'(21) }\end{array}$ & 2017/Brasil & $\begin{array}{l}\text { Acesso e acolhimento nos serviços; atendimento } \\
\text { e esclarecimento de dúvidas pelos mesmos } \\
\text { profissionais; compreensão e clareza nas } \\
\text { respostas aos doentes de TB; assistência } \\
\text { pelo profissional enfermeiro; conhecimento da } \\
\text { realidade e do contexto de vida do usuário; TDO; } \\
\text { articulação com outros serviços de saúde. }\end{array}$ & $\begin{array}{l}\text { Despreparo por parte dos profissionais de } \\
\text { enfermagem para lidar com a subjetividade dos } \\
\text { doentes; estar em situação de rua; uso de drogas } \\
\text { ilícitas; sobrecarga de trabalho dos profissionais } \\
\text { de saúde; modelo hegemônico e fragmentado } \\
\text { de saúde. }\end{array}$ \\
\hline $\begin{array}{l}\text { Wysocki et } \\
\text { al. }{ }^{(22)}\end{array}$ & 2017/ Brasil & $\begin{array}{l}\text { Descentralização das ações de controle da TB } \\
\text { na APS; orientações individuais sobre a doença } \\
\text { para a educação e empoderamento do doente } \\
\text { de TB. }\end{array}$ & $\begin{array}{l}\text { Fragilidades no envolvimento dos profissionais } \\
\text { junto às ações de controle; verticalização } \\
\text { centralizada das ações na APS; rotatividade } \\
\text { de profissionais; debilidades no processo } \\
\text { de capacitação profissional e estratégias de } \\
\text { monitoramento das ações de controle da TB; } \\
\text { desarticulação dos pontos de atenção à saúde. }\end{array}$ \\
\hline $\begin{array}{c}\text { Arakawa et } \\
\text { al. }{ }^{(23)}\end{array}$ & 2017/ Brasil & $\begin{array}{l}\text { TDO; incentivos como cestas básicas, café da } \\
\text { manhã e vale transporte; VD. }\end{array}$ & $\begin{array}{l}\text { Fragilidades no sistema público de saúde quanto } \\
\text { à assistência à saúde. }\end{array}$ \\
\hline
\end{tabular}

ACS: Agente comunitário de saúde; APS: Atenção primária à saúde; TB: Tuberculose; TDO: Tratamento diretamente observado; VD: Visitas domiciliares

\section{DISCUSSÃo}

A literatura aborda que as ações de controle da TB vêm ocorrendo de forma descentralizada no âmbito da APS ${ }^{(6,16,22)}$, e que cabe à ESF proporcionar acesso, acolhimento e assistência para os usuários, levando em conta o contexto de inserção da equipe de Saúde da Família (ESF), integrados e fundamentados nas necessidades de saúde da pessoa, família e comunidade ${ }^{(7,10,18)}$.

Isto reforça a importância da formação e do fortalecimento de vínculo e corresponsabilização - essenciais para incentivar a promoção e prevenção do agravo, bem como da adesão ao tratamento da TB, o qual deve incluir as demais dimensões que integram a perspectiva da APS: o acesso, a porta de entrada, o elenco de serviços, a coordenação, o enfoque na família, a orientação para a comunidade e a formação profissional(24); uma vez que permite aos profissionais terem uma visão integral do doente e do contexto no qual se insere, o que, associado ao TDO, possibilita a obtenção de desfechos favoráveis.

Um estudo realizado em João Pessoa, Paraíba ${ }^{(6)}$, afirma que o atributo longitudinalidade, que se relaciona com o vínculo com outros elementos, se encontra falho, uma vez que ainda ocorre a busca por serviços em outros níveis de atenção, falta de acolhimento e há existência de estigma por parte dos profissionais de saúde durante o manejo da TB, o que prejudica o estabelecimento do vínculo e a continuidade da atenção na APS.

A deficiência de constantes atualizações e capacitações é citado na literatura como um fator relacionado às dificuldades durante o manejo, a exemplo da coleta de escarro para o exame de baciloscopia, das orientações e 
da diminuição de angústias e receios por parte do doente de $\mathrm{TB}^{(7)}$, bem como dos profissionais para lidarem com a subjetividade dos doentes de $\mathrm{TB}^{(21)}$, tornando-se ferramenta imprescindível para melhorar a terapêutica e eficácia da assistência, considerando a repercussão da doença na vida do indivíduo, família e comunidade ${ }^{(10,16)}$.

Uma pesquisa(17) destaca o papel dos profissionais na linha de frente no manejo da TB na Índia, no qual, comparado ao sistema de saúde brasileiro, se assemelham ao papel dos ACS, responsáveis por estabelecerem vertentes de trabalho que vão desde o aconselhamento sobre estilo de vida, importância da continuidade do tratamento, busca ativa, acompanhamento do TDO, até o compartilhamento da prática organizativa e produção cultural da comunidade ${ }^{(15,18)}$.

Para o sucesso do tratamento, deve haver o comprometimento de toda a ESF no desempenho de atividades assistenciais, de promoção da saúde e prevenção de agravos, além da responsabilização pela saúde da comunidade(6,22), considerando o doente de TB como um ser singular e peculiar, mas sem perder de vista seu contexto familiar e social(20).

Pesquisas afirmam a importância da operacionalização do TDO para a construção de vínculo e desfecho favorável do tratamento, uma vez que essa estratégia ultrapassa a observação da ingesta medicamentosa ${ }^{(10,23)}$, permitindo uma abordagem menos fragmentada e reducionista ${ }^{(21)}$, em especial pela equipe de enfermagem, a qual destaca-se na condução do TDO por sua liderança, humanização, compromisso e ações de educação em saúde ${ }^{(18)}$, propiciando a redução do risco de transmissão da TB na comunidade ${ }^{(9)}$.

Outra estratégia para o fortalecimento do vínculo muito apontada na literatura diz respeito às VD como um momento de integração da equipe na comunidade e promoção da saúde entre os sujeitos atuantes e envolvidos no manejo para o controle da doença ${ }^{(20)}$, sendo uma maneira de (re)conhecer a realidade e o meio social do doente e sua família( ${ }^{(3)}$, facilitando, dessa forma, a responsabilização pela saúde da população das áreas adscritas ${ }^{(6)}$ e 0 estabelecimento de uma relação de confiança e reciprocidade para o manejo da doença ${ }^{(15)}$.

O vínculo e a educação em saúde são elementos complementares e primordiais para o manejo da TB por permitirem a troca de saberes, práticas e por proporcionarem mais autonomia e corresponsabilização ao doente de $\mathrm{TB}^{(9)}$. A troca mais ampla de experiências, baseada não somente nos aspectos biológicos e medicamentosos da doença, contribuem para a adesão, continuidade e sucesso do tratamento ${ }^{(14)}$.

Mesmo o diagnóstico, tratamento e acompanhamento da TB sendo oferecido gratuitamente pelo serviço público brasileiro, um estudo ${ }^{(15)}$ afirma que o manejo da doença, muitas vezes, é realizado por profissionais não capacitados, dentro de um serviço desestruturado, o que não favorece a criação do vínculo e evidencia a necessidade de um acompanhamento direto pela equipe de saúde da APS para evitar a transmissibilidade da doença e o abandono do tratamento, sendo este último um dos obstáculos para o controle da doença.

A desestruturação afeta a longitudinalidade e os outros atributos da APS, uma vez que o controle da TB está ligado à melhoria dos serviços ofertados na RAS, tais como um sistema de informação confiável, ampliação dos atendimentos descentralizados que permitem implementação do TDO, capacitação dos profissionais, fortalecimento do trabalho em equipe e otimização da referência e contrarreferência ${ }^{(7)}$ por meio de uma gestão compartilhada dos casos de TB.

Portanto, verifica-se que a operacionalização e observância das dimensões da APS, em especial o vínculo, é importante para a garantia dos resultados e da qualidade da atenção para o controle da TB, o que ultrapassa barreiras relacionadas ao envolvimento da equipe e capacitações profissionais, perpassando pela superação de fragilidades relacionadas aos aspectos organizacionais intrínsecos a diferentes cenários e serviços de saúde da RAS ${ }^{(6,22)}$.

Esta revisão possuiu como limitações a utilização de artigos em open acess, considerando que alguns resultados podem não ter sido selecionados, e a quantidade mínima de estudos realizados em outros países, em detrimento do Brasil, o que evidencia um olhar ainda fragmentado para as questões holísticas no manejo da TB. Portanto, são necessários outros estudos que incluam os resultados dos demais atributos da APS, a fim de ressignificar o conceito de vínculo.

\section{CONCLUSÃO}

Este estudo permitiu identificar elementos potencializadores e dificultadores para a construção, o estabelecimento e o fortalecimento do vínculo no manejo da tuberculose. Apontou a necessidade de novas discussões acerca da temática por meio da identificação dos pontos de estrangulamento na organização e no desempenho dos serviços de saúde, objetivando a implementação de ações integradas e intersetoriais, a partir da compreensão de aspectos que dificultam a reorientação de práticas e a oferta de serviços, tendo em vista a construção de um novo paradigma de cuidado aos doentes de tuberculose. 


\section{CONFLITOS DE INTERESSE}

As autoras afirmam que não houve conflitos de interesses na execução desta pesquisa.

\section{CONTRIBUIÇÕES}

Melisane Regina Lima Ferreira e Alexsandra Araújo Santos contribuíram com a elaboração e delineamento do estudo; a aquisição, análise e interpretação dos dados; e a redação do manuscrito. Nathalia Halax Orfão contribuiu com a aquisição, análise e interpretação dos dados; e a redação do manuscrito.

\section{FONTES DE FINANCIAMENTO}

Conselho Nacional de Desenvolvimento Científico e Tecnológico (CNPq).

\section{REFERÊNCIAS}

1. Ministério da Saúde (BR), Secretaria de Vigilância em Saúde, Departamento de Vigilância das Doenças Transmissíveis. Manual de Recomendações para o Controle da tuberculose no Brasil. Brasília: Ministério da Saúde; 2018.

2. World Health Organization. Global Tuberculosis Report 2018. Geneva: WHO; 2018.

3. Neves RR, Ferro PS, Nogueira LMV, Rodrigues ILA. Acesso e vínculo ao tratamento da tuberculose na atenção primária em saúde. Rev Pesqui (Univ. Fed. Estado Rio J). 2016;8(4):5143-9.

4. Mendes EV. As redes de atenção à saúde. $2^{\mathrm{a}}$ ed. Brasília: Organização Pan-Americana da Saúde; 2011.

5. Starfield B. Atenção primária: equilíbrio entre necessidades de saúde, serviços e tecnologia. Brasília: UNESCO; 2002.

6. Pinheiro PGOD, Sá LD, Palha PF, Oliveira RCC, Nogueira JA, Villa TCS. Pontos de estrangulamento sobre o controle da tuberculose na atenção primária. Rev Bras Enferm. 2017;70(6):1227-34.

7. Alves JCF, Paulo ZCA, Santos NMG, Pinto ESG, Davim RMB. Barreiras socioeconômico-culturais que retardam o diagnóstico da tuberculose. Rev Enferm UFPE. 2016;10(11):40210-27.

8. Orfão NH, Crepaldi NY, Brunello MEF, Andrade RLP, Monroe AA, Ruffino-Netto A, et al. Coordenação da assistência à tuberculose: registro de dados e a implementação de um sistema informatizado. Ciênc Saúde Colet. 2017;22(6):1969-77.

9. Andrade RPS, Maia VF, Queiroz RF, Carreiro GSP, Villa TCS, Pinto ESG. Contribuição dos profissionais da atenção primária à saúde para o autocuidado apoiado aos portadores de tuberculose. Rev Pesqui (Univ. Fed. Estado Rio J). 2016;8(3):4826-32.

10. Figueiredo TMRM, Pinto ML, Cardoso MAA, Silva VA. Desempenho no estabelecimento do vínculo nos serviços de atenção à tuberculose. Rev Rene. 2011;12(esp):1028-35.

11. Melnyk BM, Fineout-Overholt E. Making the case for evidence-based practice and cultivating a spirit of inquiry. Philadelphia: Lippincot Williams \& Wilkins; 2011. Capítulo 1, Evidence-based practice in nursing \& healthcare: a guide to best practice; p. 3-24.

12. Lewis CP, Newell JN. Improving tuberculosis care in low income countries - a qualitative study of patients understanding of "patient support" in Nepal. BMC Public Health. 2009;9(190).

13. Chhea C, Warren N, Manderson L. Health worker effectiveness and retention in rural Cambodia. Rural Remote Health. 2010;10(3):1391.

14. Ponce MAS, Vendramini SHF, Santos MR, Santos MLSG, Scatena LM, Villa TCS. Vínculo profissional/doente no tratamento da tuberculose: desempenho da atenção básica em município do interior paulista. Rev LatinoAm Enferm. 2011;19(5).

15. Rocha DDS, Adorno RDCF. Abandono ou descontinuidade do tratamento da tuberculose em Rio Branco, Acre. Saúde Soc. 2012;21(1):232-45. 
16. Marquieviz J, Alves IS, Neves EB, Ulbricht LA. Estratégia de Saúde da Família no controle da tuberculose em Curitiba (PR). Ciênc Saúde Colet. 2013;18(1):265-71.

17. Kielmann K, Datye V, Pradhan A, Rangan S. Balancing authority, deference and trust across the publicprivate divide in health care: tuberculosis health visitors in western Maharashtra, India. Glob Public Health. 2014;9(8):975-92.

18. Souza KMJS, Sá LD, Silva LMC, Palha P. Atuação da Enfermagem na transferência da política do tratamento diretamente observado da tuberculose. Rev Esc Enferm USP. 2014;48(5):874-82.

19. Balderrama P, Vendramini SF, Santos MLSG, Ponce MAZ, Oliveira IC, Villa TCS, et al. Porta de entrada para o diagnóstico da tuberculose: avaliação da estrutura dos serviços. Rev Eletrônica Enferm. 2014;16(3):511-9.

20. Clementino FS, Miranda FAN. Tuberculose: acolhimento e informação na perspectiva da visita domiciliária. Rev Enferm UERJ. 2015;23(3):350-4.

21. Furlan MCR, Santos AG Jr, Marcon SS. O vínculo com o profissional de saúde no tratamento de tuberculose: percepção dos usuários. Rev Enferm Centro-Oeste Mineiro. 2017;7(e1934).

22. Wysocki $A D$, Ponce $M A Z$, Brunello $M E F$, Beraldo $A A$, Vendramini SHF, Scatena $L M$, et al. Atenção Primária à Saúde e tuberculose: avaliação dos serviços. Rev Bras Epidemiol. 2017;20(1):161-75.

23. Arakawa T, Magnabosco GT, Andrade RLP, Brunello MEF, Monroe AA, Ruffino-Netto A, et al. Programa de controle da tuberculose no contexto municipal: avaliação de desempenho. Rev Saúde Pública. 2017;51(23):1-9.

24. Gomes ALC, Sá LD. As concepções de vínculo e a relação com o controle da tuberculose. Rev Esc Enferm USP. 2009;43(2):365-72.

\author{
Endereço para correspondência: \\ Melisane Regina Lima Ferreira \\ Fundação Universidade Federal de Rondônia - UNIR \\ BR 364, Km 9,5 \\ CEP: 76801-059 - Porto Velho - RO - Brasil \\ E-mail: melisane1206@gmail.com
}

Como citar: Ferreira MRL, Santos AA, Orfão NH. O vínculo no tratamento da tuberculose na atenção primária à saúde: uma revisão integrativa. Rev Bras Promoç Saúde. 2019;32:9540. 